Pesq. Vet. Bras. 36(11):1109-1115, novembro 2016 DOI: $10.1590 / \mathrm{S} 0100-736 \mathrm{X} 2016001100009$

\title{
Fauna helmintológica de catetos (Tayassu tajacu Linnaeus, 1758) procedentes da Amazônia Brasileira ${ }^{1}$
}

\author{
Ronaldo A. Pereira-Junior ${ }^{2 *}$, Sebastiana A.P. Sousa ${ }^{2}$, Marcelo C. Oliveira ${ }^{2}$, Alana de \\ A. Valadares ${ }^{2}$, Estevam G.L. Hoppe ${ }^{3}$ e Katyane S. Almeida ${ }^{2}$
}

\begin{abstract}
Pereira-Junior R.A., Sousa S.A.P., Oliveira M.C., Valadares A.A., Hoppe E.G.L. \& Almeida K.S. 2016. [Helminthic parasites of the collared peccaries (Tayassu tajacu Linnaeus, 1758) of the Brazilian Amazon.] Fauna helmintológica de catetos (Tayassu tajacu Linnaeus, 1758) procedentes da Amazônia Brasileira. Pesquisa Veterinária Brasileira 36(11):1109-1115. Escola de Medicina Veterinária e Zootecnia, Universidade Federal do Tocantins, BR-153 Km 112, Zona Rural, Caixa Postal 132, Araguaína, T0 77804-970, Brazil. E-mail: pereirajunior.ra@outlook.com

Tayassu tajacu, popularly known as collared peccary, is a wildlife species found throughout the American continent, being abundant in their places of occurrence. This study aimed to describe the helminthological fauna, as well as the infection indicators, of the collared peccary (T. tajacu) coming from the Brazilian Amazon. Five adult peccaries (three males and two females) were captured in the Araguaína region, Tocantins, Brazil, with the aid of traps. The collared peccaries were reassured, chemically restrained and euthanized according to the Brazilian Good Practice Guide for Animal Euthanasy of the Federal Board of Veterinary Medicine. The species found were identified and the infection indicators were determined (infection rate, abundance, mean intensity and intensity variation). A total of 1394 helminths of nine nematode species were collected: Eucyathostomum dentatum, Cruzia brasiliensis, Monodontus semicircularis, Monodontus aguiari, Spiculopteragia tayassui, Texicospirura turki, Parabronema pecariae, Physocephalus sexalatus and Cooperia punctata. Helminths with higher percentages of infection were Physocephalus sexalatus, Parabronema pecariae and Monodontus aguiari found in $100 \%$ of the examined animals; the latter was the most abundant (164) and had a highest mean intensity (164) and total number of helminths (820). In contrast, Spiculopteragia tayassui presented lower indicators, found only one female in this study. In this studythe the first occurrence of Cruzia brasiliensis parasitizing collared peccary (T. Tajacu) in Brazil was reported, specie hitherto described and only found parasitizing domestic pigs.
\end{abstract}

INDEX TERMS: Cruzia brasiliensis, Helminths, Tayassuidae, peccaries, Tayassu tajacu.

RESUMO.- Tayassu tajacu, popularmente conhecido como cateto, é uma espécie silvestre encontrada em quase todo o continente Americano, sendo abundante em seus locais de ocorrência. 0 trabalho objetivou conhecer a fauna helmintológica, bem como os indicadores de infecções, de catetos

\footnotetext{
${ }^{1}$ Recebido em 3 de fevereiro de 2016.

Aceito para publicação em 28 de julho de 2016.

${ }^{2}$ Escola de Medicina Veterinária e Zootecnia, Universidade Federal do Tocantins, Campus Universitário de Araguaína, BR-153 Km 112, Cx. Postal 132, Zona Rural, Araguaína, T0 77804-970, Brasil.

${ }^{3}$ Faculdade de Ciências Agrárias e Veterinárias, Universidade Estadual Paulista "Júlio de Mesquita Filho" (Unesp), Via de Acesso Prof. Dr. Paulo Donato Castellane s/n, Zona Rural, Jaboticabal, SP 14887-900, Brasil. *Autor para correspondência: pereirajunior.ra@outlook.com
}

(T. tajacu) procedentes da Amazônia Brasileira. Para tanto, foram utilizados cinco animais adultos (três machos e duas fêmeas), capturados no município de Araguaína, estado do Tocantins, com auxílio de armadilhas. Os catetos foram tranquilizados, contidos quimicamente e eutanasiados segundo o Guia Brasileiro de Boas Práticas para Eutanásia em Animais do Conselho Federal de Medicina Veterinária. As espécies encontradas foram identificadas e os indicadores de infecção foram determinados (percentual de infecção, abundância, intensidade média e variação da intensidade). Foi coletado um total de 1394 helmintos, sendo nove espécies de nematódeos: Eucyathostomum dentatum, Cruzia brasiliensis, Monodontus semicircularis, Monodontus aguiari, Spiculopteragia tayassui, Texicospirura turki, 
Parabronema pecariae, Physocephalus sexalatus, Cooperia punctata. Os helmintos com maiores percentuais de infecção foram Physocephalus sexalatus, Parabronema pecariae e Monodontus aguiari, encontrados em 100\% dos animais examinados, sendo este último o mais abundante (164), com maior intensidade média (164) e com maior número total de helmintos (820). Em contrapartida, Spiculopteragia tayassui foi a espécie que obteve menores indicadores, sendo encontrada apenas uma fêmea neste estudo. Foi relatada, neste trabalho, a primeira ocorrência de Cruzia brasiliensis parasitando catetos (T. tajacu) no Brasil, espécie até então descrita e encontrada apenas parasitando suínos domésticos.

TERMOS DE INDEXAÇÃO: Cruzia brasiliensis, helmintoses, Tayassuidae, catetos, Tayassu tajacu.

\section{INTRODUÇÃO}

Tayassu tajacu, popularmente conhecido como cateto, pertence à ordem Artiodactyla, à Sub-ordem Nonruminantia, Superfamília Suidea e à Família Tayassuidae (Nowak 1999). Possui ampla distribuição geográfica que vai desde o sul dos Estados Unidos até o norte da Argentina (Margarido \& Mangini 2001, Sonner et al. 2004).

Além de ser uma importante fonte de proteínas para subsistência em várias regiões no interior do Brasil, principalmente em populações indígenas e ribeirinhas (Smith 1976, Mandujano \& Rico-Gray 1991, Redford 1992, Parry et al. 2009, Almeida et al. 2011), a carne destes animais é bastante apreciada em grandes centros urbanos no país e no exterior, principalmente na Europa (Nogueira-Filho \& Nogueira 2000). A criação de catetos em cativeiro vem crescendo para possibilitar a exploração econômica da carne destes animais que, além de muito saborosa, apresenta um baixo teor de gordura e é rica em ácidos graxos insaturados (Freire-Lopes et al. 2007, Albuquerque et al. 2009, Santos et al. 2009).

Para Mota et al. (2003), o conhecimento da epidemiologia das parasitoses, bem como a avaliação da relação parasito-hospedeiro, são fundamentais para o estabelecimento de um sistema de controle efetivo de helmintoses. A falta destas informações faz com que os tratamentos antihelmínticos utilizados em programas de controle de parasitoses sejam, na maioria das vezes, ineficazes, levando ao surgimento de casos clínicos da doença e, consequentemente, perdas produtivas nos criadouros (Pereira-Junior et al. 2014). Estas perdas estão relacionadas com o retardo na produção, os custos com tratamentos e profilaxia e, em casos extremos, o óbito dos animais parasitados (Macrae 1993, Perry \& Randolph 1999, Almeida et al. 2008).

Em 1986 foi realizado um levantamento parasitológico em catetos na região central da Amazônia, onde observaram o parasitismo nestes animais por oito espécies de helmintos, sendo elas: Monodontus semicircularis, Eucyathostomum dentatum, Texicospirura turki, Parabronema pecariae, Gongylonema sp., Trichuris sp. e Moniezia benedeni (Neto \& Thatcher 1986)

No compêndio de Vicente et al. (1997) foram descritas espécies de nematódeos parasitando catetos no Brasil, en- tre elas estão Dirofilaria acutiuscula, E. dentatum, M. semicircularis, Gongylonema baylisi, Nematodirus molini e Oesophagostomum dentatum.

Em um trabalho realizado por Nascimento (2004), foram encontrados 5661 helmintos parasitando 10 catetos do Pantanal do Mato Grosso do Sul, dividindo-se em 19 espécies diferentes: P. sexalatus, Ascarops strongylina, $P$. pecariae, T. turki, Trichostrongylus axei, Spiculopteragia tayassui, Cooperia espatulata, Cooperia punctata, Parostertagia heterospiculum, Trichostrongylus colubriformes, Strongyloides ransomi, Monodontus aguari, M. semicircularis, Macracanthorhyncus hirudinaceus, Oesophagostomum dentatum, E. dentatum, Stichorchis giganteus, Metastrongylus salmi e D. acutiuscula.

Apesar de existirem alguns trabalhos relatando parasitos de T. tajacu, ainda são necessários mais estudos acerca dos parasitos que acometem esta espécie, sobretudo na região Amazônica, uma região ainda pouco explorada neste sentido. Desta forma, conhecer a helmintofauna de catetos da Amazônia brasileira, bem como os indicadores de infecção, é de fundamental importância pela contribuição à parasitologia, além de auxiliar diretamente para criação desses animais em cativeiro, fornecendo dados que servem como base para estabelecimento de tratamento e prevenção mais racionais.

Em função desta problemática, este trabalho objetivou identificar as espécies de nematódeos encontradas em catetos procedentes da região de Araguaína, Estado do Tocantins, e avaliar a relação parasito-hospedeiro por meio de indicadores parasitológicos de infecção.

\section{MATERIAL E MÉTODOS}

0 presente trabalho foi realizado conforme orientações da Comissão de Ética no Uso de Animais da Universidade Federal do Tocantins (CEUA-UFT) sob protocolo no 23101.003939/2012-35, e autorização do IBAMA no 42.253-1. Para tanto, foram capturados cinco catetos (Tayassu tajacu) adultos, sendo três machos e duas fêmeas, procedentes da zona rural do município de Araguaína, Estado do Tocantins (latitude $07^{\circ} 11^{\prime} 28^{\prime \prime}$ sul e longitude $48^{\circ} 12^{\prime} 26^{\prime \prime}$ oeste), localizado a uma altitude de $227 \mathrm{~m}$ e distante cerca de $368 \mathrm{~km}$ da capital, Palmas. Segundo Köppen-Geiger, o clima na região é classificado como Aw (Clima tropical com chuvas de verão), com temperaturas que variam entre $20^{\circ} \mathrm{C} \mathrm{e} 36^{\circ} \mathrm{C}$ ao longo do ano, com temperatura média anual de $27^{\circ} \mathrm{C}$. A precipitação pluviométrica anual varia entre $9 \mathrm{~mm}$ em julho, e $281 \mathrm{~mm}$ em março, com média de $142 \mathrm{~mm}$ e com precipitação anual acima de $1.700 \mathrm{~mm}$ (INMET 2014).

A captura foi realizada utilizando armadilhas confeccionadas com madeira para esta finalidade (Neri 2004), as quais foram instaladas em propriedades particulares de criação extensiva de bovinos, sendo que cada um dos animais foi capturado em diferentes propriedades próximas ao município de Araguaína. Após a captura, os animais foram tranquilizados com acepromazina na dose de $0,2 \mathrm{mg} / \mathrm{kg}$ via intramuscular (IM) e contidos quimicamente com a utilização de cloridrato de quetamina na dose de $20 \mathrm{mg} /$ $\mathrm{kg}$, também via IM. Em seguida foram eutanasiados por meio da administração de tiopental sódico 2,5\%, por via endovenosa, até que fosse atingido o coma barbitúrico. Posteriormente, administrou-se cloreto de potássio $19,1 \%$ até a obtenção de parada cardíaca, obedecendo as recomendações da Resolução CFMV no 1000/2012 (Brasil 2012) e do Guia Brasileiro de Boas Práticas 
para a Eutanásia em Animais do Conselho Federal de Medicina Veterinária (CFMV 2012).

Os animais foram necropsiados a campo com a separação de cada segmento anatômico do trato digestório (esôfago, estômago, intestino delgado e intestino grosso) com barbante para impedir a passagem de conteúdo de um segmento para o outro. Cada segmento foi aberto e lavado separadamente com água corrente em bandeja metálica para total remoção e coleta do conteúdo que, em seguida, foi tamisado em peneiras com abertura numérica de $100 \mu \mathrm{m}$. Também foram observados traqueia, coração, pulmão, fígado, pâncreas, rins e tecido subcutâneo para pesquisa de helmintos.

Os conteúdos resultantes, após a lavagem, foram fixados em solução de Railliet e Henry de acordo com a técnica preconizada por Travassos (1950), envasados e enviados ao Laboratório de Higiene e Saúde Pública da Escola de Medicina Veterinária e Zootecnia, Universidade Federal do Tocantins, para identificação das espécies.

Os helmintos presentes nos conteúdos foram coletados com auxílio de um microscópio estereoscópico. Foram então separados por gênero e sexo, contados e mantidos em solução de Railliet \& Henry para posterior identificação quanto à espécie.

A identificação das espécies foi realizada seguindo a metodologia descrita por Travassos (1950) para a classificação de nematódeos: os nematódeos encontrados foram retirados do fixador (Railliet e Henry) e colocados, por 30 minutos, em ácido acético 80\% para clarificação, a fim de possibilitar a observação das formações celulares. Para os nematódeos maiores ou para observação dos espículos em machos, onde a clarificação apenas com ácido acético não foi suficiente, foi utilizado o creosoto de Faya, após o ácido acético, para uma melhor diafanização e clarificação do parasito.

Os parasitos foram identificados de acordo com Travassos (1929, 1937a, 1937b), Costa (1965), Chitwood \& Cordero de Campillo (1966), Vicente et al. (1997), Vicente et al. (2000), Nascimento (2004), Araújo (2011).

Utilizou-se, de acordo com Bush et al. (1997), a estatística descritiva baseada nos helmintos para a determinação dos indicadores de infecção (percentual de infecção, abundância, intensidade média, variação de intensidade).

\section{RESULTADOS}

Nos cinco animais capturados foram coletados e identificados um total de 1394 helmintos distribuídos no estômago, intestino delgado e intestino grosso, que foram classifica- dos em 10 diferentes espécies, porém uma delas, devido à presença apenas de fêmeas, foi classificada apenas até a superfamília (Trichostrongyloidea). A maior quantidade de helmintos foi encontrada no intestino delgado, totalizando 959 parasitos, seguido pelo estômago com 240 nematódeos e, com menor quantidade, o intestino grosso com 195 helmintos.

As espécies observadas neste estudo foram: Parabronema pecariae, Physocephalus sexalatus, Texicospirura turki e Spiculopteragia tayassui no estômago; Cooperia punctata, Monodontus aguiari, Monodontus semicircularis e espécie não identificada da superfamília Trichostrongyloidea no intestino delgado; e Cruzia brasiliensis e Eucyathostomum dentatum no intestino grosso. Os dados de indicadores de infecção como intensidade média, variação da intensidade, abundância, percentual de infecção e o total de helmintos, estão expressos no Quadro 1. Não foram encontrados helmintos parasitando outras vísceras ou tecido subcutâneo dos animais estudados. Também não foram observados cestódeos ou trematódeos.

Com relação aos indicadores de infecção (Fig.1), foi observado que Physocephalus sexalatus, Parabronema pecariae e Monodontus aguiari apresentaram o maior percentual de infecção, sendo encontrados em 100\% dos animais estudados. Texicospirura turki e Spiculopteragia tayassui apresentaram as menores prevalências, sendo observados em apenas um animal cada (20\%).

A intensidade média variou entre 1 e 164, sendo observada a maior para Monodontus aguiari (164), seguida por Texicospirura turki (120) e Cruzia brasiliensis (82). As menores intensidades médias observadas foram para Spiculopteragia tayassui (1) e para Eucyathostomum dentatum $(8,67)$.

Com relação à abundância, Monodontus aguiari, Cruzia brasiliensis e Texicospirura turki foram os mais abundantes, apresentando $164,32,8$ e 24 , respectivamente, e os que apresentaram maior quantidade em número total de helmintos sendo 820, 169 e 120, respectivamente. A menor abundância foi observada em Spiculopteragia tayassui $(0,2)$ e Eucyathostomum dentatum $(5,2)$, que também foram os que apresentaram menor número total de helmintos, 1 e 26 , respectivamente.

Quadro 1. Indicadores das infecções naturais de helmintos observados nos segmentos gastrointestinais de cinco catetos (Tayassu tajacu), procedentes da região de Araguaína, Tocantins

\begin{tabular}{|c|c|c|c|c|c|c|c|}
\hline \multirow[t]{2}{*}{ Espécies } & \multirow[t]{2}{*}{ Habitat } & \multicolumn{6}{|c|}{ Indicadores das infecções } \\
\hline & & $\begin{array}{l}\text { Variação da } \\
\text { intensidade }\end{array}$ & $\begin{array}{l}\text { Intensidade } \\
\text { média }\end{array}$ & Abundância & $\begin{array}{l}\text { Percentual } \\
\text { de infecção }\end{array}$ & $\begin{array}{c}\text { Total de } \\
\text { helmintos }\end{array}$ & $\%$ \\
\hline Parabronema pecariae & Estômago & $02-36$ & $14,6(5)^{*}$ & 14,6 & 100 & 73 & 5,24 \\
\hline Physocephalus sexalatus & Estômago & $03-21$ & $9,2(5)$ & 9,2 & 100 & 46 & 3,30 \\
\hline Texicospirura turki & Estômago & $0-120$ & $120(1)$ & 24 & 20 & 120 & 8,61 \\
\hline Spiculopteragia tayassui & Estômago & $0-1$ & $1(1)$ & 0,2 & 20 & 1 & 0,07 \\
\hline Cooperia punctata & Int. delgado & $0-49$ & $29(3)$ & 17,4 & 60 & 87 & 6,25 \\
\hline Monodontus aguiari & Int. delgado & $10-308$ & $164(5)$ & 164 & 100 & 820 & 58,82 \\
\hline Monodontus semicircularis & Int. delgado & $0-21$ & $15(3)$ & 9 & 60 & 45 & 3,23 \\
\hline Trichostrongyloidea & Int. delgado & $0-7$ & 7 (1) & 1,4 & 20 & 7 & 0,50 \\
\hline Cruzia brasiliensis & Int. grosso & $0-164$ & $82(2)$ & 32,8 & 40 & 169 & 12,12 \\
\hline Eucyathostomum dentatum & Int. grosso & $0-13$ & $8,67(3)$ & 5,2 & 60 & 26 & 1,86 \\
\hline Total de helmintos & - & - & - & - & - & 1394 & 100,00 \\
\hline
\end{tabular}

$\overline{()^{*}=\text { Número de animais positivos, \% }}=$ em relação ao número total de helmintos. 


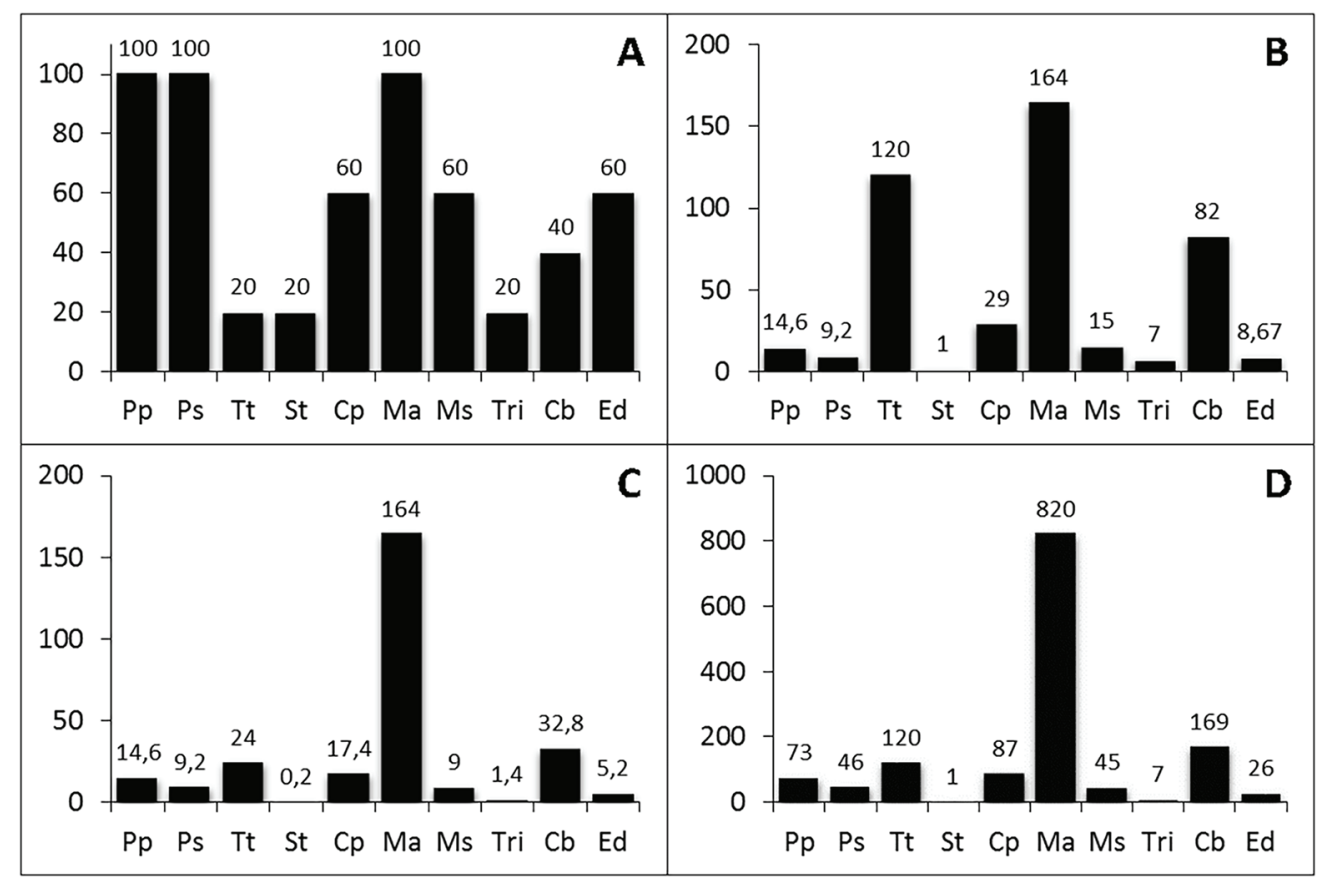

Fig.1. Percentual de infecção (A), intensidade média (B), abundância (C) e número total de helmintos (D) observados em cinco catetos (Tayassu tajacu) adultos, procedentes da região da Amazônia Brasileira, município de Araguaína, Estado do Tocantins. (Pp = Parabronema pecariae $; \mathrm{Ps}=$ Physocephalus sexalatus; $\mathrm{Tt}=$ Texicospirura turki; $\mathrm{St}=$ Spiculopteragia tayassui; $\mathrm{Cp}=$ Cooperia punctata; $\mathrm{Ma}=$ Monodontus aguiari; Ms = Monodontus semicircularis; Tri = Trichostrongyloidea; $\mathrm{Cb}=$ Cruzia brasiliensis; Ed = Eucyathostomum dentatum .

Apesar de algumas espécies de parasitos, como $M$. aguiari, apresentarem abundância e intensidade média de parasitismo alta, não foram observadas lesões macroscópicas nos órgãos examinados neste estudo.

\section{DISCUSSÃO}

Neste estudo foram encontradas nove espécies de helmintos de seis famílias diferentes, sendo todas elas de nematódeos, o que está de acordo com Brandão et al. (2009), que relataram que os mamíferos silvestres se apresentam parasitados, geralmente, em maior quantidade por nematódeos que cestódeos ou trematódeos, sendo estes às vezes nem são encontrados em estudos parasitológicos. Igualmente, Nascimento (2004) observou que espécies de nematódeos perfaziam cerca de $90 \%$ do total de helmintos encontrados em seu estudo.

No entanto, Travassos et al. (1969), Vicente et al. (1997) e Nascimento (2004) relacionam a presença de trematódeos em catetos, pela observação de Stichorchis giganteus, porém não foram encontrados trematódeos parasitando catetos no presente estudo helmintológico. Isto pode ser devido à localização geográfica e condições ambientais nas quais foi realizado o presente estudo, pois este parasito está mais frequente em áreas alagadiças. Isto porque Stichorchis giganteus tem como hospedeiro intermediário um gênero de caramujos aquáticos e que estão presentes em regiões alagadiças, como é o caso, por exemplo, do pantanal dos Paiaguás, local de captura de animais nos estudos de Nascimento (2004).

Das espécies de nematódeos observadas nos resultados deste estudo parasitando catetos (Tayassu tajacu), apenas Cruzia brasiliensis (Fig.2) ainda não foi relatada parasitan- do estes animais no Brasil (Vicente et al. 1997, Nascimento 2004), sendo uma espécie descrita e relatada somente em suínos domésticos (Costa 1965, Vicente et al. 1997); fazendo com que este estudo seja o primeiro relato de C. brasiliensis parasitando catetos. Isto reforça a possibilidade de os catetos atuarem como reservatórios de parasitos para outras espécies, como os suínos, podendo representar um problema em granjas, caso não haja o controle de acesso de animais silvestres às proximidades das instalações. Da mesma forma, em suínos criados em sistema semi-extensivo, a problemática é ainda maior, uma vez que o contato com catetos pode acontecer com maior facilidade. No entanto, o inverso também pode ser importante, uma vez que suínos domésticos criados soltos podem levar parasitos para populações de catetos no ambiente antes livre deste parasito e, desta forma, fazer com que os animais se infectem e possam vir a óbito, dependendo do grau de infecção.

Neto \& Thatcher (1986), ao realizarem um estudo preliminar de helmintos em catetos, revelaram a presença de sete espécies de nematódeos e uma de cestódeo na Região Amazônica Central. Semelhantes a este estudo e aos realizados por Nascimento et al. (1996) e Nascimento (2004), os autores observaram a presença de $M$. semicircularis, $E$. dentatum, T. turki, P. sexalatus e P. pecariae.

Vicente et al. (1997) ao listar os parasitos de T. tajacu, relacionou apenas seis helmintos parasitando esta espécie, sendo elas Dirofilaria acutiuscula, Eucyathostomum dentatum, Gongylonema baylisi, Monodontus semicircularis, Nematodirus molini e Oesophagostomum dentatum, o que muito difere deste estudo, uma vez que apenas duas das nove espécies encontradas estão relacionadas parasitando catetos neste compêndio. 


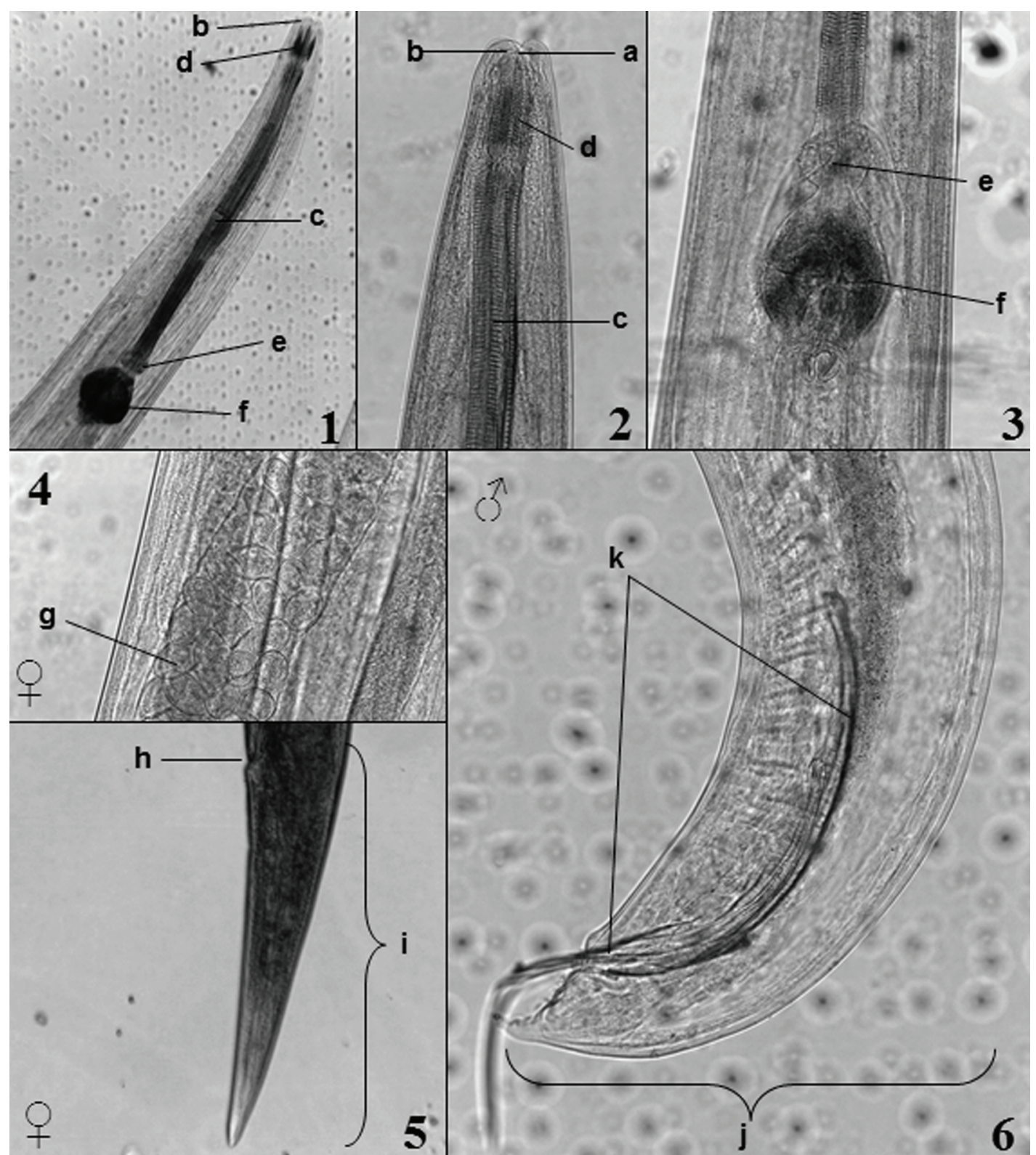

Fig.2. Cruzia brasiliensis (Fêmeas = 10,01-14,24mm; Machos = 9,60-13,76mm). (1 e 2) Extremidade anterior, (3) dilatação no final do esôfago e bulbo, (4) útero da fêmea evidenciando os ovos no seu interior, 5) extremidade posterior da fêmea, (6) extremidade posterior do macho ( $\mathrm{a}$ = abertura oral, $\mathrm{b}=$ boca trilabiada com lábios subtriangulares, $\mathrm{c}=$ esôfago oxiuriforme longo $(0,93-2,51 \mathrm{~mm}$ de comprimento), $d=$ faringe contendo tres fileiras de ganchos, $e=$ dilatação da porção final do esôfago, $f=$ bulbo $(0,29-0,40 \mathrm{~mm} x$ 0,27-0,35mm), $g$ = ovos relativamente grandes $(0,1 \times 0,06 \mathrm{~mm})$, com casca espessa e rugosa dentro do útero, $\mathrm{h}=$ ânus, $\mathrm{i}=$ cauda cônica com ponta delgada, $\mathrm{j}$ = cauda cônica com asas caudais ausentes, $\mathrm{k}=$ espículos iguais e filiformes com cerca de $0,8-0,93 \mathrm{~mm}$ ). $0 b j .4 \mathrm{x}$ (1 e 5$)$ e obj.10x $(2,3,4$ e 6$)$.

No trabalho realizado por Nascimento (2004), foram encontradas 19 espécies de helmintos parasitando catetos, sendo que algumas não foram observadas no presente estudo: D. acutiuscula parasitando o tecido subcutâneo; $M e$ tastrongylus salmi parasitando pulmões; S. giganteus, Oesophagostomum dentatum parasitando o intestino grosso; Macracanthorhyncus hirudinaceus, Strongyloides ransomi, Trichostrongylus colubriformis, Parostertagia heterospiculum, Cooperia spatulata parasitando intestino delgado; e Trichostrongylus axei e Ascarops strongylina parasitando o estômago. Todavia, a presença de T. turki não foi observada por este autor, diferindo deste trabalho.
As diferenças de parasitismo observadas entre os estudos podem ser devidas às diferenças ambientais nos locais de captura dos animais, isto porque Tayassu tajacu pode ser encontrado em diversos tipos de hábitats, estando desde locais muito secos até áreas alagadiças. Isto influencia, por exemplo, a observação de trematódeos que, em função de sua biologia, são encontrados em grande quantidade em áreas alagadiças. A proximidade dos pontos de captura com criações de animais domésticos também pode ser um fator determinante para a variação da carga parasitária, uma vez que estes animais podem servir de fonte de infecção para catetos. 
Com relação aos indicadores de infecção, os valores mais expressivos de abundância e prevalência foram observados em Monodontus aguiari, que também apresentou maior intensidade média e maior número total de helmintos. Estes resultados diferem daqueles obtidos por Nascimento (2004) no Brasil e Samuel \& Low (1970) no Texas, que observaram maiores indicadores de infecção por $P a$ rostertagia heterospiculum.

As espécies de espirurídeos, T. turki e P. sexalatus, desempenharam papel importante nos indicadores de infecção, sendo observada a terceira maior abundância e intensidade média para T. turki e uma prevalência de $100 \%$ para $P$. sexalatus. Os espirurídeos requerem um hospedeiro intermediário para o seu desenvolvimento, em particular coleópteros coprófagos, onde surgem as formas infectantes (Levine 1980). Os hábitos alimentares de catetos são muito diversificados, indo desde frutas e sementes, até insetos e pequenos vertebrados (Margarido \& Mangini 2001), bem como algumas espécies de anelídeos (Sicuro 1996). Estes hábitos alimentares apresentados por T. tajacu são, provavelmente, a principal causa dos importantes indicadores de infecção observados para os espirurídeos, pois, ao ingerirem coleópteros coprófagos contendo larvas infectantes, estes animais se infectam e/ou aumentam sua carga parasitária. Se assemelhando a este estudo, Samuel \& Low (1970) e Corn et al. (1985) nos EUA e Neto \& Tatcher (1986) no Brasil, observaram altos índices para T. turki e P. sexalatus em catetos. Entretanto, ao avaliar os indicadores de infecções de espirurídeos, Nascimento (2004) observou índices importantes de abundância e prevalência para $P$. sexalatus e A. strongylina.

Monodontus aguiari foi a espécie que apresentou valores maiores para todos os indicadores de infecções (abundância, percentual de infecção, intensidade média, variação da intensidade e número total de helmintos). Este ancilostomatídeo foi descrito por Travassos (1937b) em cutias (Dasyprocta agouti) e, segundo Nascimento (2004), a via de infecção desse nematódeo se dá pelo contato da larva infectante com a pele, embora também possa haver transmissão transplacentária e a lactotransmissão. Portanto, em razão da estrutura social elaborada pelos catetos, que formam grupos e são gregários no período noturno, essa forma de infecção pode contribuir para os altos indicadores de infecções observados em M. aguiari.

Autores como Graber et al. (1964), Prestwood et al. $(1975,1976)$ e Nascimento (2004), comentam sobre as possibilidades de infecções cruzadas de helmintos entre espécies de animais silvestres e domésticos. Graber et al. (1964), ao estudarem Bovidae e Suidae silvestres, encontraram 47 espécies de helmintos, das quais 26 necessitam de hospedeiro específico e 21 são comuns aos animais domésticos e silvestres, levando os autores a questionarem o potencial destes animais silvestres como reservatórios de parasitos para animais domésticos que convivem no mesmo ambiente.

Segundo Nascimento (2004), a presença de C. spatulata, C. punctata, T. columbriformis e T. axei parasitando catetos, evidencia a ocorrência de infecção cruzada entre animais domésticos e silvestres, uma vez que a provável origem desses parasitos para os catetos sejam os bovinos. A presença de $C$. brasiliensis, observada neste estudo, apenas reforça a ideia da ocorrência destas infecções cruzadas entre animais domésticos e silvestres, pois esta espécie foi descrita e até hoje encontrada apenas parasitando suínos domésticos, sendo estes a provável fonte de infecção para os catetos.

Um fator que pode atuar como agravante é a concentração de animais domésticos e silvestres em áreas restritas de pastagens, próximas às vazantes, que ocorrem no período seco do ano, o que leva ao aumento da contaminação ambiental e, consequentemente, o aumento da tensão de contaminação. Essas condições são adequadas para o desenvolvimento, a sobrevivência de formas imaturas do parasito e de hospedeiros intermediários no ambiente. Além disso, as condições facilitam a transmissão das infecções causadas por helmintos, principalmente nos animais silvestres (Nascimento 2004).

Portanto, os resultados do presente estudo demonstram que os cinco exemplares de Tayassu tajacu capturados na região de Araguaína/TO, encontravam-se parasitados por nove espécies de nematódeos, sendo: Parabronema pecariae, Physocephalus sexalatus, Texicospirura turki, Spiculopteragia tayassui, Cooperia punctata, Monodontus aguiari, Monodontus semicircularis, Cruzia brasiliensis, Eucyathostomum dentatum. Os maiores indicadores de infecção foram encontrados em M. aguiari, que apresentou maior intensidade média, abundância e número total de helmintos. As maiores porcentagens de infecção foram observadas em $M$. aguiari, P. pecariae e P. sexalatus. Apesar de algumas espécies apresentarem altos indicadores de infecção, nenhum dos animais estudados apresentou sinais clínicos ou lesões macroscópicas causados pelo parasitismo, o que não significa que este parasitismo não cause problemas e perdas em criações comerciais. Este trabalho, além de fornecer dados básicos e fundamentais sobre o parasitismo de catetos na região norte do Estado do Tocantins, relatou, pela primeira vez, a presença de Cruzia brasiliensis parasitando T. tajacu, espécie até então encontrada parasitando apenas suínos domésticos. Isto evidencia a problemática da criação de animais domésticos em contato com animais silvestres, reforçando ainda mais a importância deste tipo de estudo. Este tipo de conhecimento é fundamental para que, em criações comerciais de catetos, o controle e tratamento de helmintoses possa ser efetivo e de modo racional.

Agradecimentos.- Ao professor Dr. Sandro Estevan Moron, da Universidade Federal do Tocantins (UFT) pelo apoio prestado para a aquisição de imagens e medidas dos parasitos. A José Hairton Tebaldi da Universidade Estadual Paulista Júlio de Mesquita Filho (Unesp) por importante ajuda na identificação de espécies de nematódeos. Ao Conselho Nacional de Desenvolvimento Científico e Tecnológico - CNPq pela concessão de bolsa de mestrado, e à Universidade Federal do Tocantins pela concessão de uma bolsa de iniciação científica (PIBIC-UFT).

\section{REFERÊNCIAS}

Albuquerque N.I., Contreras C.C., Alencar S., Meirelles C.F., Aguiar A.P., Moreira J.A. \& Packer I.U. 2009. Propriedades da carne e perfil de ácidos graxos do pernil de catetos (Tayassu tajacu) alimentados com torta de babaçu (Orbignya phalerata). Arq. Bras. Med. Vet. Zootec. 61:1419-1427. 
Almeida A.M.B., Nogueira-Filho S.L.G., Nogueira S.S.C. \& Munhoz A.D. 2011. Aspectos hematológicos de catetos (Tayassu tajacu) mantidos em cativeiro. Pesq. Vet. Bras. 31:173-177.

Almeida K.S., Freitas F.L.C., Tebaldi J.H. \& Nascimento A.A. 2008. Helmintos parasitos de mocós (Kerodon rupestris Rodentia: Caviidae) de vida livre e de cativeiro, no semi-árido nordestino brasileiro. Arch. Vet. Sci. 13:133-139.

Araújo L.R.F. 2011. Descrição taxonômica de Cruzia sp. nov. e Aspidodera sp. nov. (Nematoda, Ascaridida), parasitas de intestino grosso de Philander opossum Linnaeus, 1758, marsupial de Carajás-Pará, Brasil. Dissertação de Mestrado em Biologia de Agentes Infecciosos e Parasitários, Instituto de Ciências Biológicas, Universidade Federal do Pará, Belém, PA. 102p.

Brandão M.L., Chame M., Cordeiro J.L.P. \& Chaves S.A.M. 2009. Diversidade de helmintos intestinais em mamíferos silvestres e domésticos na Caatinga do Parque Nacional Serra da Capivara, Sudeste do Piauí, Brasil. Revta Bras. Parasitol. Vet. 18:19-28.

Brasil 2012. Resolução №1000, de 11 de maio de 2012. Dispõe sobre procedimentos e métodos de eutanásia em animais e dá outras providências. Diário Oficial da União, Brasília, 17 de maio de 2012. Seção 1, p.124-125.

Brites-Neto J. \& Thatcher V.E. 1986. Estudos parasitológicos preliminares em taiassuídeos (Tayassu tajacu) na Amazônia Central. Revta Bras. Med. Vet. 8:175-178.

Bush A.O., Lafferty K.D., Lotz J.M. \& Shostaki A.W. 1997. Parasitology meets ecology on its own terms: Margolis et al. revisited. J. Parasitol. 83:575583.

CFMV 2012. Guia Brasileiro de Boas Práticas em Eutanásia em Animais: conceitos e procedimentos recomendados. Vol.1. Conselho Federal de Medicina Veterinária, Brasília. 62p.

Chitwood M.B. \& Cordero de Campillo M. 1966. Texicospirura turki gen. et sp. n. (Nematoda: Spiruroidea) from the stomach of the peccary in the United States, and a key to the genera of Ascaropsinae. J. Parasitol. 52:307-310.

Corn J.L., Pence D.B. \& Warren R.J. 1985. Factors affecting the helminth community strutura of adult collared peccaries in southern Texas. J. Wildl. Dis. 21:254-263.

Costa H.M.A. 1965 Cruzia brasiliensis n. sp. (Nematoda-Cruziidae) parasite de Sus domesticus. Arq. Esc. Vet. UFMG 17:61-69.

Freire-Lopes K.R., Bezerra F.J., Monteiro-Nogueirall C., Barreto Júnior R.A. \& Veras De Paula V. 2007. Teores de colesterol e ácidos graxos em carne de catetos (Tayassu tajacu) criados em cativeiro. Revta Caatinga 20:6975 .

Graber M., Doutre M., Finelle P., Keravec J., Ducroz G. \& Nokotencar P. 1964. Les helmintes de quelques artiodactyles sauvages appartenant aux familles de bovidés et dês suides. Ces mammiféres, em Républica du Tchad et en R.C.A. soint.ls des réservoirs de parasites, pour les animaux domestiques vivant à leur contact? Rev. Élev. Méd. Vét. Pays Trop. 17:377-421.

INMET (Instituto Nacional de Meteorologia), 2014. Ministério da Agricultura, Pecuária e Abastecimento. Dados meteorológicos. Disponível em: <http://www.inmet.gov.br>. Data de acesso: 02/01/2014.

Levine N.L. 1980. Nematode Parasites of Domestic Animals and Man. 2nd ed. Burgess Publishing Co., Minneapolis. 417p.

Macrae J.C. 1993. Metabolic consequences of intestinal parasitism. Proc. Nutr. Soc. 52:121-130.

Mandujano S. \& Rico-Gray V. 1991. Hunting, use and knowledge of the biology of the white-tailed deer (Odocoileus virginianus Hays) by the maya of central Yucatan, Mexico. J. Ethnobiol. 11:175-183.

Margarido T.C.C. \& Mangini P.R. 2001. Order Artiodactyla, Family Tayassuidade (Peccaries), p.377-391. In: Fowler M.E. \& Cubas Z.S. (Eds), Biology, Medicine and Surgery of South American Wild Animals. State University Press, Ames, Iowa.

Mota M.A., Campos A.K. \& Araújo J.V. 2003. Controle biológico de helmintos parasitos de animais: estágio atual e perspectivas futuras. Pesq. Vet. Bras. 23:93-100.
Nascimento A.A. 2004. Infecções naturais por helmintos parasitos de artiodáctilos, no estado do Mato Grosso do Sul (Pantanal de Paiaguás), Brasil. Tese de Livre-Docência, Faculdade de Ciências Agrárias e Veterinárias, Universidade Estadual Paulista, Jaboticabal, SP. 70p.

Nascimento A.A., Bonuti M.R., Mapeli E.B., Tebaldi J.H. \& Arantes I.G. 1996. Helmintos parasitos de suínos (Sus scrofa domesticus), cateto (Tayassu tajacu) e veado catingueiro (Mazama gouazoubira). Anais 15을 Congresso Panamericano de Ciências Veterinárias, Campo Grande, MS, p.79. (Resumo)

Neri F.M. 2004. Ecologia e conservação de catetos, Tayassu tajacu (Artiodactyla, Tayassuidea) em áreas do Nordeste do Estado de São Paulo. Tese (Doutorado em Ciências), Centro de Ciências Biológicas e da Saúde, Universidade Federal de São Carlos, São Carlos, SP. 148p.

Nogueira-Filho S.L.G. \& Nogueira S.S.C. 2000. Criação comercial de animais silvestres: Produção e comercialização da carne e subprodutos na região sudeste do Brasil. Revta Econ. Nordeste 31:188-195.

Nowak R.M. 1999. Walker's Mammals of the World. 6th ed. Johns Hopkins University Press, Maryland. 2015p.

Parry L., Barlow J. \& Peres C.A. 2009. Hunting for sustainability in tropical secondary forests. Conserv. Biol. 23:1270-1280.

Pereira-Junior R.A., Sousa S.A.P., Valadares A.A., Oliveira M.C. \& Almeida K.S. 2014. Fauna helmintológica de catetos (Tayassu tajacu Linnaeus, 1758) e suas implicações para a produção comercial: revisão de literatura. Revta Cient. Eletr. Med. Vet. 12(23):1-22.

Perry B.D. \& Randolph T.F. 1999. Improving the assessment of the economic impact of parasitic diseases and their control in production animals. Vet. Parasitol. 84:145-168.

Prestwood A.K., Kellogg F.E., Pursglove S.R. \& Hayes F.A. 1975. Helminths parasitisms among intermingling insular populations of white-tailed deer, feral cattle and feral swine. J. Am. Vet. Med. Assoc. 166:787-790.

Prestwood A.K., Pursglove S.R. \& Hayes F.A. 1976. Parasitism among white-tailed deer and domestic sheep on common range. J. Wildl. Dis. 12:380-500.

Redford K.H. 1992. The empty forest. BioScience 42:412-422.

Samuel W.M. \& Low W.A. 1970. Parasites of the collared peccary from Texas. J. Wildl. Dis. 6:16-23.

Santos D.O., Mendes A., Nogueira S. \& Nogueira-Filho S.L.G. 2009. Criação comercial de caititus (Pecari tajacu): uma alternativa para o agronegócio. Revta Bras. Saúde Prod. Anim. 10:1-10.

Sicuro F.L. 1996. Interferências acerca da coexistência de Taiassuídeos e Suídeos ferais (Mammalia, Artiodactyla) no Pantanal de Nhecolândia (MS): um modelo ecomorfológico. Dissertação de Mestrado em Ciências Biológicas (Zoologia), Universidade Federal do Rio de Janeiro, Rio de Janeiro, RJ. 162p.

Smith N.J.H. 1976. Utilization of game along Brasil's transamazon highway. Acta Amazonica 6:455-466.

Sonner J.B., Miglino M.A., Santos T.C., Carvalhal R., Assis-Neto A.C., Moura C.E.B. \& Oliveira M.F. 2004. Aspectos macroscópicos e morfométricos dos testículos em catetos e queixadas. Biota Neotrop. 4:1-13.

Travassos L. 1929. Sobre o Monodontus semicircularis (Molin, 1861). Revta Museu Paulista 16:867-879.

Travassos L. 1937a. Eucyathostomum dentatum Molin, 1861 (Nematoda: Strongyloidea). Mem. Inst. Oswaldo Cruz 32:95-100.

Travassos L. 1937b. Gênero Monodontus Molin, 1861 (Nematoda: Strongyloidea). Mem. Inst. Oswaldo Cruz 32:225-231.

Travassos L. 1950. Introdução ao estudo da helmintologia. Revta Bras. Biol. 169p.

Travassos L., Freitas J.F.T. \& Kohn A. 1969. Trematódeos do Brasil. Mem. Inst. Oswaldo Cruz 67:1-886.

Vicente J.J., Muniz-Pereira L.C., Noronha D. \& Pinto R.M. 2000. Description of males of Parabronema pecariae Ivaschkin, 1960 (Nematoda, Habronematoidea) parasitizing Peccaries (Mammalia, Tayassuidae) in Brazil. Mem. Inst. Oswaldo Cruz 95:849-851.

Vicente J.J., Rodrigues H.O., Gomes D.C. \& Pinto R.M. 1997. Nematóides do Brasil. Parte V: Nematóides de mamíferos. Revta Bras. Biol. 14:452p. 